\title{
Evaluation of mucosal healing in Small Bowel Crohn's disease treated with Certolizumab Pegol assessed by wireless capsule endoscopy
}

\author{
Ira Shafran, Patricia Burgunder*, Renee DePanicis, Kara Fitch, Sally Hewit and Laurie Abbott
}

Shafran Gastroenterology Center, Winter Park, FL, USA

\begin{abstract}
Background: Certolizumab Pegol, a pegylated anti-TNF agent, is currently approved to reduce the signs and symptoms of Crohn's Disease (CD) and maintain clinical response in adult patients with moderately to severely active $\mathrm{CD}$ who have had inadequate response to conventional therapy. Biologic agents have demonstrated efficacy in the healing of gut mucosa leading to better long-term outcomes by sustaining steroid free remission, decreasing the need for major surgery and hospitalizations, and by improving patients overall quality of life. Prior studies have revealed certolizumab pegol induced endoscopically measured clinical response and remission at 10 and 54 weeks. Our primary objective was to evaluate mucosal healing assessed by wireless capsule endoscopy (WCE) using the Lewis scoring system (LS) in patients with moderate-to-severe small bowel Crohn's Disease (SBCD) treated with certolizumab pegol.

Methods: We performed a prospective, single center, open-label trial in 15 patients with documented moderate-to-severe SBCD for a period of six months. All patients were randomized to receive standard induction therapy $(400 \mathrm{mg}$ ) with certolizumab pegol at 0,2 , and 4 weeks, then standard maintenance dose therapy (400 $\mathrm{mg}$ ) every 4 weeks through the end of the 28 week study period. Wireless capsule endoscopy with LS was performed at baseline, 12 weeks, and 24 weeks. Blood work, including complete metabolic panel (CMP), hemogram (HEMGPD), and C-reactive protein (CRP) were obtained as well as Crohn's Disease Activity Index (CDAI) and Short Inflammatory Bowel Disease Questionnaire (SIBDQ) at baseline and at every visit throughout the study period.

Results: Eighteen patients underwent screening assessment with 15 patients qualifying to participate and 13 patients completing the trial. Of the 15 intent to treat patients, 6 were male and 9 were female with an average age of 33 (range 21-50) and a mean disease duration of 11.2 (range 1-26) years. Twelve patients had no prior $\mathrm{CD}$ surgery and 3 had previous small bowel resection. Ten of the 15 enrolling patients were secondary non-responders (SNR) to biologics; 4 lost response to both infliximab and adalimumab, 6 lost response to either infliximab or adalimumab, 3 were biologically naïve, and 2 had an allergic reaction to infliximab. None of the 10 biologically exposed patients were primary non-responders to other agents.

Ten of the 13 patients (77\%) who completed this trial demonstrated an overall improvement in mucosal healing, CDAI, and SIBDQ. Of those who responded to certolizumab pegol, the mean LS fell from 1663 at baseline to 226 at 24 weeks and the mean CDAI decreased from 256 at baseline to 140 at 28 weeks. The mean SIBDQ increased in responders from 40 at baseline to 51 by the end of study.

Conclusions: This study demonstrates evidence of mucosal healing using certolizumab pegol in patients with moderate-to-severe SBCD. This study establishes a proof of concept that WCE used in conjunction with the LS is a valuable diagnostic test to assess mucosal healing in patients with SBCD treated with certolizumab pegol. Certolizumab Pegol was well-tolerated in this population with no safety issues. Larger, placebo-controlled trials are warranted to assess small bowel mucosal healing in $\mathrm{CD}$ patients treated with certolizumab pegol.
\end{abstract}

\section{Introduction}

\section{Crohn's disease and mucosal healing}

Crohn's disease $(\mathrm{CD})$ is a chronic inflammatory bowel disorder that can affect any part of the gastrointestinal tract. In approximately $30 \%-40 \%$ of patients it involves the small bowel, most commonly the terminal ileum, but lesions proximal to the terminal ileum are often present [1-3]. The goal of medical treatment for CD includes improving patients' quality of life while reducing complications of disease including hospitalizations and surgery [4]. Current medical treatments and past studies have defined response to treatment by measuring clinical symptoms of response and remission without consistently including mucosal healing endpoints. With the current use of diseasemodifying medications such as certolizumab pegol, mucosal healing has emerged as an increasingly important goal of therapy [3]. Mucosal healing, or endoscopic remission, is associated with increased rates of clinical remission, fewer hospitalizations and abdominal surgeries, and increased work productivity with improved quality of life for patients
$[2,3]$. There is a growing realization that these goals of treatment translate into an overall reduction of the cost-burden of CD [5-11].

\section{Certolizumab pegol}

The pro inflammatory cytokine tumor necrosis factor $\alpha(\mathrm{TNF} \alpha)$ is highly expressed in the blood, colonic tissue, and stool of patients with Crohn's disease $[1,3,4]$. Infliximab and adalimumab are engineered IgG1 monoclonal antibodies that bind to TNFa (the first represents a chimeric molecule and the latter has been derived from human origin) and are effective in the induction and maintenance of response

Correspondence to: Patricia Burgunder, Shafran Gastroenterology Center, 701 West Morse, BLVD, Winter Park, FL 32789, USA Tel: 407-629-5869; Fax: 407629-7250; E-mail: Pburgunder@me.com

Received: November 03, 2016; Accepted: November 16, 2016; Published: November 21, 2016 
and remission in patients with $\mathrm{CD}$. The efficacy of infliximab in the treatment of $\mathrm{CD}$ has been attributed to multiple mechanisms, including reverse signaling through membrane-bound TNFa and the induction of apoptosis of T cells and monocytes [1,3,4]. Certolizumab Pegol, a pegylated humanized Fab1 fragment of an anti-TNFa monoclonal antibody, has several characteristics that differentiate it from infliximab and adalimumab. In vitro, certolizumab pegol has a higher affinity for $\mathrm{TNF} \alpha$, is devoid of the Fc portion of the antibody, and does not induce complement activation, antibody-dependent cellular cytotoxicity, or apoptosis [3,4].

Induction treatment with certolizumab pegol has been shown to be effective for the treatment of moderately-to-severely active Crohn's disease using a $400 \mathrm{mg}$ dose of certolizumab pegol every 4 weeks after induction in patients with a serum CRP level of at least $10 \mathrm{mg}$ per liter. Response rates at week 12 were significantly higher than those in the placebo group [4].

\section{Wireless capsule endoscopy and lewis score}

Wireless capsule endoscopy was approved by the FDA in 2001 and has gained acceptance as providing state-of-the-art endoscopic imaging of the small intestine [12-14]. Capsule endoscopy is now commonly used in the evaluation of patients with obscure gastrointestinal bleeding including iron deficiency anemia, suspected and known CD, malabsorption syndromes, (e.g. celiac disease) and chronic abdominal pain. Imaging (CT and MR enterography) assists in proximal SBCD assessment, and can provide insight in identifying active mucosal inflammation and importantly identifies fibrostenotic stricturing disease, but it lacks ability to directly visualize mucosa. Because terminal Ileal intubation can be elusive during colonoscopy and visualization of more proximal lesions are not possible on direct exam, WCE offers great ability to visualize small bowel ulcers often missed by other imaging modalities. Our center has previously published safety data on 98 patients with SBCD undergoing a total of 148 separate WCE studies without a single capsule retention event underscoring the safety and utility of WCE testing. A meta-analysis comparing WCE to other imaging modalities of the small bowel for inflammatory bowel disease has established that WCE has an incremental diagnostic yield of 25\%$40 \%$ over that of other modalities [13]. Despite these investigations, there is a lack of standardization when describing these lesions in terms of their extent and severity. Specifically, no unified language of findings has been developed and no severity scale of mucosal disease activity or even a threshold for disease diagnosis has been agreed upon [12]. The Lewis score was developed to specifically quantify SBCD objectively with parameters and descriptors of inflammatory change identified. The goal of this scoring system was to assure that the final numerical score reflected the global assessment and that the global assessment agreed with the ranking of finding severity. Results were compiled for the three categories: 1) no or clinically insignificant change, 2) mild change, and 3) moderate or severe change. The scoring index is useful in measuring small bowel mucosal disease activity on WCE with the final index including three parameters: 1.) villous edema, 2.) ulcer and 3.) stenosis. A score $<135$ is designated normal or clinically insignificant mucosal inflammatory change, a score between 135 and 790 is mild, and a score $\geq 790$ is moderate to severe [12].

\section{Methods}

\section{Study design}

Our study was a single center, prospective, open label 28 week trial conducted between April 2010 and October 2013. The protocol was approved by the Western Institutional Review Board on January 5, 2010 (registration number NCT01053559). After meeting inclusion/ exclusion criteria, all patients were assigned to receive open-label subcutaneous injections of certolizumab pegol at a dose of $400 \mathrm{mg}$ at weeks 0,2 , and 4 and then every 4 weeks. They were followed through week 28. Patients completed CDAI and SIBDQ scoring at each visit. At weeks $0,2,4,8,12,16,20,24$ and 28, patients were evaluated in the clinic. Data was collected from diaries kept by patients, adverse events and concomitant medications were recorded, and laboratory tests were performed. Wireless capsule endoscopy was performed at baseline, week 12 , and week 24 . To reduce inter-observer variation, a single experienced reader was utilized and Lewis score evaluation calculated.

\section{Patients}

All patients were identified though our community practice population with a known history of SBCD. Those who presented to the office with a flare of SBCD during the study period and met all eligibility criteria, were provided with and willingly gave their consent. Eligible patients were adults aged 18-70 with confirmed CD that included the small bowel and naive to certolizumab pegol. All participants had active CD with a CDAI score of 220 to 450, WCE performed at baseline, and a qualifying LS of 135 or greater. Concomitant medications were allowed prior to enrollment in the study and included: Two week stable doses of steroids (oral or ano-rectal); 4 week stable doses of 5-aminosalicylates (5-ASA's), antibiotics, anti-diarrheals, analgesics, probiotics, and 8 week stable doses of immunosuppressants. Patients having any and all contraindications for the use of certolizumab pegol including tuberculosis, hepatitis, infection, abscess, malignancy, congestive heart failure, demylelinating neurological conditions, and cytopenia were excluded from participation. Patients with a contraindication for the use of WCE including small bowel obstruction or stricture were also excluded. Previous treatment with certolizumab pegol, pregnancy or lactation, or any clinically significant abnormal lab values that would pose a risk to the subject while receiving certolizumab pegol were excluded.

\section{Primary endpoints}

Our primary objective was to assess the efficacy of certolizumab pegol in healing small bowel CD ulcers by utilizing WCE and the Lewis Scoring System in subjects with moderately to severely active small bowel Crohn's disease. Our secondary objectives were to assess the efficacy of certolizumab pegol in the induction of clinical remission and the maintenance of remission to 26 weeks, to assess the impact of certolizumab pegol on the SIBDQ score, and to assess the safety of certolizumab pegol.

\section{Results}

Eighteen patients underwent screening assessment, with 15 patients qualifying to participate and 13 patients completing the trial (Figure 1). Of the 15 intent to treat patients, 6 were male and 9 were female with an average age of 33 (range 21-50) and a mean disease duration of 11.2 (range 1-26) years. Twelve patients had no prior CD surgery and 3 had previous small bowel resection. Ten of the 15 patients were secondary non-responders (SNR) to biologics, 4 lost response to both infliximab and adalimumab, 6 lost response to either infliximab or adalimumab, 3 were biologically naïve, and 2 had an allergic reaction to infliximab. None of the 10 biologically exposed patients were primary nonresponders to other agents (Table 1). Ten of the 13 patients (77\%) who completed this trial demonstrated an overall clinical improvement in mucosal healing, CDAI, and SIBDQ (Figure 2) of those who responded 


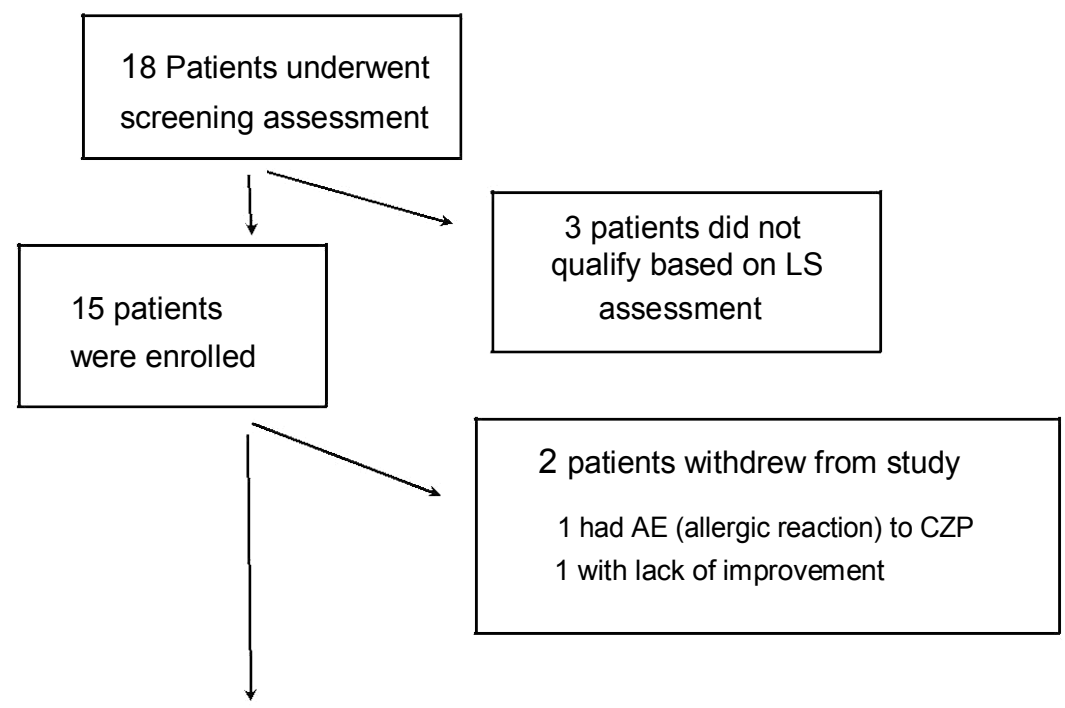

13 patients completed study to week 28

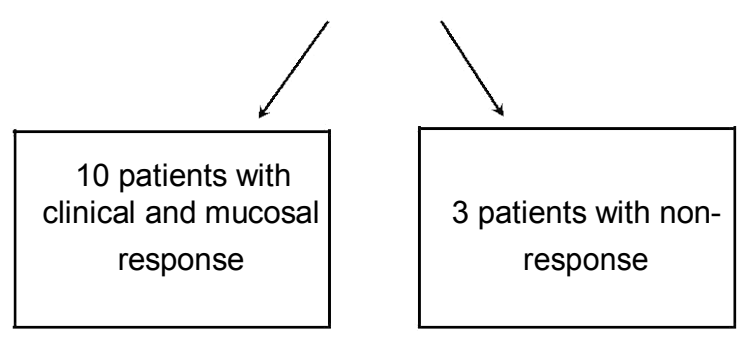

Figure 1. Enrollment.

Table 1. Characteristics of the Patients in the Intention-to-Treat Population $(\mathrm{N}=15)$.

\begin{tabular}{|c|c|}
\hline Mean age in years (range) & $32(21-50)$ \\
\hline \multicolumn{2}{|l|}{$\operatorname{Sex}(n, \%)$} \\
\hline Male & $6(40 \%)$ \\
\hline Female & $9(60 \%)$ \\
\hline \multicolumn{2}{|l|}{ Duration of disease, years } \\
\hline Mean & 11.2 \\
\hline Median & 9 \\
\hline Range & $1-26$ \\
\hline Current smoker (n,\%) & $1(7 \%)$ \\
\hline \multicolumn{2}{|l|}{ Disease site (n,\%) } \\
\hline Terminal ileum & $15(100 \%)$ \\
\hline \multicolumn{2}{|l|}{ Prior anti-TNF therapy $(\mathrm{n}, \%)$} \\
\hline Biologic Naive & $3(20 \%)$ \\
\hline One prior agent & $8(53 \%)$ \\
\hline Two prior agents & $4(27 \%)$ \\
\hline Prior resection (n,\%) & $3(20 \%)$ \\
\hline
\end{tabular}

to certolizumab pegol, the mean LS fell from 1663 at baseline to 226 at 24 weeks and the mean CDAI decreased from 256 at baseline to 140 at 26 weeks. The mean SIBDQ increased in responders from 40 at baseline to 51 by the end of study (Figure 3). Two enrolled patients were unable to complete this trial. The first had a history of prior allergic reaction to infliximab and experienced a cutaneous allergic reaction to the first induction dose of certolizumab pegol significant enough to discontinue treatment. The reaction was treated with a short course of oral steroids and quickly resolved without sequelae. A second patient, biologically naive and on 6-MP, received 5 doses of certolizumab pegol. The patient participated in this trial until week 12 when the second WCE study revealed non-healing (LS unchanged at 3040). Due to worsening symptom scores and disease activity, the patient was withdrawn from participation.

\section{Discussion}

Acknowledging the limitations of our small sample size and open label design, our study offers valuable proof of concept data using 

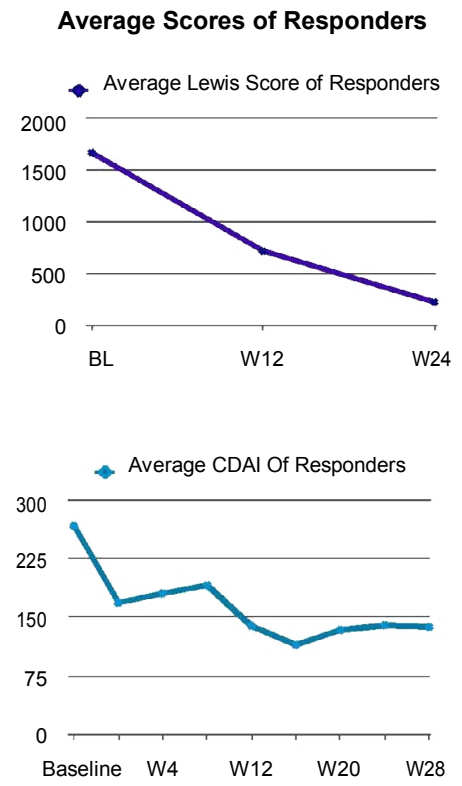

- Average SIBDQ Scores of Responders

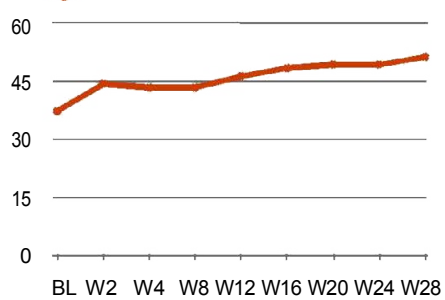

Figure 2. Average Scores of Responders.

certolizumab pegol and assessing for mucosal healing with WCE and the Lewis scoring system. We have shown that patients with advanced Crohn's disease who have failed multiple prior biologic and immunosuppressant therapies responded both clinically and endoscopically to certolizumab pegol. Prospective research focused on whether targeted therapies achieve mucosal healing, and if, in fact, mucosal healing does positively impact $\mathrm{CD}$ disease activity and quality of life while reducing utilization of healthcare resources beyond 6 months is warranted.

\section{Funding support}

Grant support provided by UCB, Inc

\section{References}

1. Sandborn WJ, Feagan BG, Stoinov S, Honiball PJ, Rutgeerts P, et al. (2007) Certolizumabpegol for the treatment of Crohn's disease. $N$ Engl J Med 357: 228-238. [Crossref]

2. Schreiber S, Khaliq-Kareemi M, Lawrance IC, Thomsen OØ, Hanauer SB, et al. (2007) Maintenance therapy with certolizumabpegol for Crohn's disease. N Engl J Med 357: 239-250.[Crossref]

3. Hébuterne X, Lémann M, Bouhnik Y, Dewit O, Dupas JL, et al. (2013) Objective To evaluate the efficacy of CertolizumabPegol (CertolizumabPegol) in improving endoscopic lesions in patients with active ileocolonicCrohn's disease (CD). Gut 62:201-208. [Crossref]

4. Hanauer SB, Sandborn W; Practice Parameters Committee of the American College of Gastroenterology (2001) Management of Crohn's disease in adults. Am J Gastroenterol96: 635-643.[Crossref]

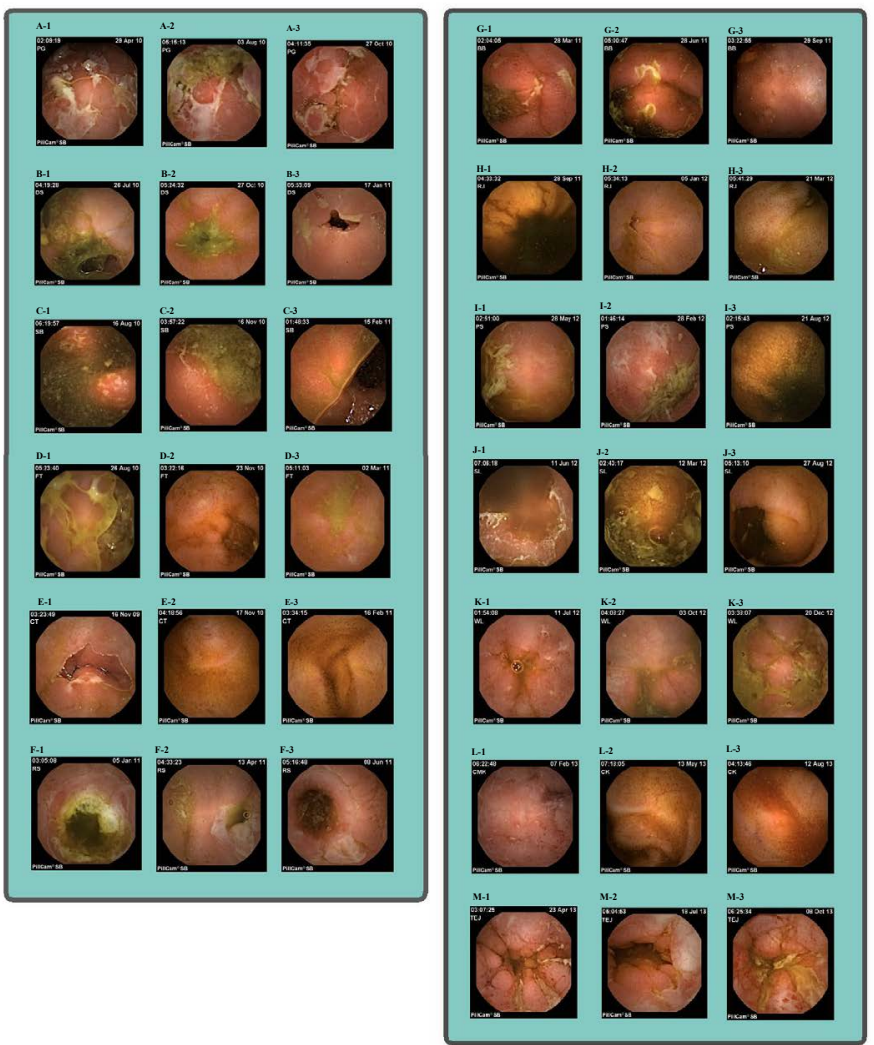

Figure 3. Small Bowel Capsule Endoscopy images in 13 patients studied (A-M), at 3 time points: 1-Baseline, 2-Week 12, 3-Week 24. Images represent the worst areas of involvement and are compared at the same location according to capsule localizer (transit time of each capsule will vary).

Patient A reveals no evidence of mucosal healing; Patient $\mathbf{B}$ with clear improvement in depth and number of fissures; Patient $\mathbf{C}$ with reduction of edema and healing of superficial ulcers; Patient D with resolution of edema, fissures and improvement in number of aphthous ulcers; Patient $\mathbf{E}$ with complete healing of edema and ulcers; Patient $\mathbf{F}$ with clear evidence of healing; Patient $\mathbf{G}$ with decreased edema and improvement in fissures; Patient $\mathbf{H}$ with significant reduction in ulceration, edema; Patient $\mathbf{I}$ without evidence of endoscopic healing, circumferential edema and ulceration; Patient $\mathbf{J}$ with evidence of complete healing, resolution of aphthous ulceration and edema;

Patient $\mathbf{K}$ with mild baseline disease and evidence of resolution; Patient $\mathbf{L}$ with healing of superficial ulcerations.; Patient M w

5. Baert F, Moortgat L, Van Assche G, Caenepeel P, Vergauwe P, et al. (2010) Mucosal Healing Predicts Sustained Clinical Remission in Patients With Early-Stage Crohn's Disease. Gastroenterology 138: 463-468. [Crossref]

6. Gralnek IM, Defranchis R, Seidman E, Leighton JA, Legnani P, et al. (2008) Development of a capsule endoscopy scoring index for small bowel mucosal inflammatory change. Aliment Pharmacol Ther 27: 146-154.[Crossref]

7. Rutgeerts P, Vermeire S, Van Assche G (2007) Mucosal healing in inflammatory bowel disease: impossible ideal or therapeutic target? Gut 56: 453-455.[Crossref]

8. Efthymiou A, Viazis N, Mantzaris G, Papadimitriou N, Tzourmakliotis D, et al. (2008) Does clinical response correlate with mucosal healing in patients with Crohn's disease of the small bowel? A prospective, case-series study using wireless capsule endoscopy. Inflamm Bowel Dis 14: 1542-1547. [Crossref]

9. Pineton de Chambrun G, Peyrin-Biroulet L, Lémann M, Colombel JF (2010) Clinical implications of mucosal healing for the management of IBD. Nat Rev GastroenterolHepatol7: 15-29.[Crossref]

10. vanAssche G, Vermeire S, Rutgeerts P (2010) Mucosal healing and anti TNFs in IBD Curr Drug Targets 11: 227-233.[Crossref]

11. Neurath MF, Travis SP (2012) Mucosal healing in inflammatory bowel diseases: a systematic review. Gut61: 1619-1635.[Crossref]

12. Gralnek IM, Defranchis R, Seidman E, Leighton JA, Legnani P, et al. (2008) Development of a capsule endoscopy scoring index for small bowel mucosal 
Shafran I (2016) Evaluation of mucosal healing in Small Bowel Crohn's disease treated with Certolizumab Pegol assessed by wireless capsule endoscopy

inflammatory change. Aliment Pharmacol Ther 27: 146-154.[Crossref]

13. Efthymiou A, Viazis N, Mantzaris G, Papadimitriou N, Tzourmakliotis D, et al. (2008) Does Clinical Response Correlate with Mucosal Healing n Patients with Crohn's
Disease of the Small Bowel? A Prospective, Case-series Study Using Wireless Capsule Endoscopy. Inflamm Bowel Dis 14:1542-1547. [Crossref]

14. Shafran I, Burgunde P, Kwa M (2013) The Use of Wireless Capsule Endoscopy in Community Practice: An 11 Year Experience. Inflammatory Bowel Diseases 19.

Copyright: $@ 02016$ Shafran I. This is an open-access article distributed under the terms of the Creative Commons Attribution License, which permits unrestricted use, distribution, and reproduction in any medium, provided the original author and source are credited. 